\title{
Nonadiabatic quantum molecular dynamics with hopping. II. Role of nuclear quantum effects in atomic collisions
}

\author{
M. Fischer, J. Handt, and R. Schmidt* \\ Institut für Theoretische Physik, Technische Universität Dresden, D-01062 Dresden, Germany
}

(Received 30 April 2014; published 29 July 2014)

\begin{abstract}
The role of electron-nuclear correlations, i.e., quantum effects in the nuclear motion in atomic collisions with complex targets, is discussed using the recently developed nonadiabatic quantum molecular dynamics with hopping method [Fischer, Handt, and Schmidt, paper I of this series, Phys. Rev. A 90, 012525 (2014)]. It is shown that the excitation process is nearly unaffected by electron-nuclear correlations as long as integral quantities are considered (total kinetic energy loss), whereas the relaxation mechanism of the molecular target is greatly affected (total fragmentation probability). To describe highly differential quantities (kinetic energy loss as a function of the scattering angle), however, the consideration of nuclear quantum effects during the initial excitation process is indispensable, even in collisions where one would expect purely classical behavior of the nuclei due to their small de Broglie wavelength. The calculations reproduce and explain in detail old but still unexplained experimental data of differential energy-loss spectroscopy in $\mathrm{He}+\mathrm{He}$ and $\mathrm{He}+\mathrm{H}_{2}$ collisions.
\end{abstract}

DOI: 10.1103/PhysRevA.90.012526

PACS number(s): 31.15.xv, 34.50.Gb, 31.15.ee, 36.40.-c

\section{INTRODUCTION}

Recently, much progress has been made in the understanding of nonadiabatic phenomena, arising from electronic excitations in collisions between complex particles. In particular, collisions of atoms or ions with molecules and clusters have attracted much attention in various fields such as material science, plasma physics, astrophysics, and biophysics. Nowadays, ion-beam radiation provides a fundamental resource for cancer therapy and is largely utilized to modify mechanical, electronic, and magnetic properties of nanostructures [1-5].

Refined scattering experiments have allowed us to gain detailed insights into the complex mechanisms and the interplay between electronic and vibrational excitations involved in these collisions. Thereby, a rich variety of interesting phenomena has been uncovered, for example, transparency in collisions involving water clusters [6] and fullerenes [7], charge transfer in ion-molecule [8-11] and ioncluster collisions [12], nonadiabatic fusion between fullerenes $[13,14]$, and (multi)fragmentation processes in collisions involving metal clusters [15], hydrocarbons [16,17], or fullerenes [18-20].

The theoretical description of nonadiabatic collisions is a challenging task, as it requires the self-consistent treatment of all nuclear and electronic degrees of freedom. The (in principle, necessary) solution of the time-dependent Schrödinger equation for electrons and nuclei is a priori precluded due to the invincible computational effort. Instead, solely mixed quantum-classical methods have been developed for this purpose, e.g., grid-based [21-25] or basis expansion-based [26-29] ab initio methods using time-dependent densityfunctional theory (TD-DFT) for the electrons. Thereby, the nuclear motion is described purely classically, whether selfconsistently in the mean-field limit (also known as Ehrenfest dynamics in quantum chemistry [30] or simply as meanfield approximation in solid-state physics [31]) or by using the straight-line approximation [32-34] (widely applied in

*Ruediger.Schmidt@tu-dresden.de ion-atom collision theory). In both cases, electron-nuclear correlations (i.e., quantum effects in the nuclear dynamics) are neglected completely. Alternatively, Babikov et al. [35] have developed an interesting nonadiabatic molecular-dynamics (MD) method that accounts approximately for electronnuclear correlations in collisions. Its microscopic foundation, however, is less general, as it builds upon an approximate treatment of the electron dynamics in terms of the so-called diatomics in molecule approach [36]. Here we report an ab initio approach, which combines electron dynamics with quantum correlated nuclear motion, in atomic and molecular collisional physics.

The nonadiabatic quantum molecular dynamics with hopping (NA-QMD-H) method, developed recently [37] (cited as Paper I in the following), provides such an approach by coupling TD-DFT or time-dependent Hartree-Fock theory in a basis expansion to trajectory surface hopping dynamics for the nuclei. In Paper I we outlined the general formalism as a rigorous extension of the general [26-29] and confirmed [15,38-40] NA-QMD method. We demonstrated exemplarily the specific relevance of electron-nuclear correlations for the simplest possible (two-state) collision model of the $\mathrm{H}^{+}+\mathrm{H}$ system. It was shown that for this benchmark system the NA-QMD-H approach exactly reproduces the full quantummechanical results. In the present paper we apply the whole many-body version of the NA-QMD-H formalism (without laser field) and elaborate the general role of electron-nuclear correlations (ENCs) in complex atomic collisions.

In Sec. II we present a systematic study of various atom-molecule and atom-cluster collisions (with increasing electronic and nuclear complexity) as a function of the impact velocity $v$. For the sake of completeness, the velocity $v$ is varied in a wide range, i.e., starting from $v \ll 1$ a.u. (where the collisions proceed adiabatically, i.e., only vibrational excitations occur) up to velocities $v \lesssim 1$ a.u. (where the mechanism is strongly nonadiabatic, i.e., initially exclusively electronic excitations appear). The generic influence of ENCs on the excitation and relaxation mechanism will be examined by studying integral scattering observables, in particular, the total kinetic energy loss of the atomic projectile 


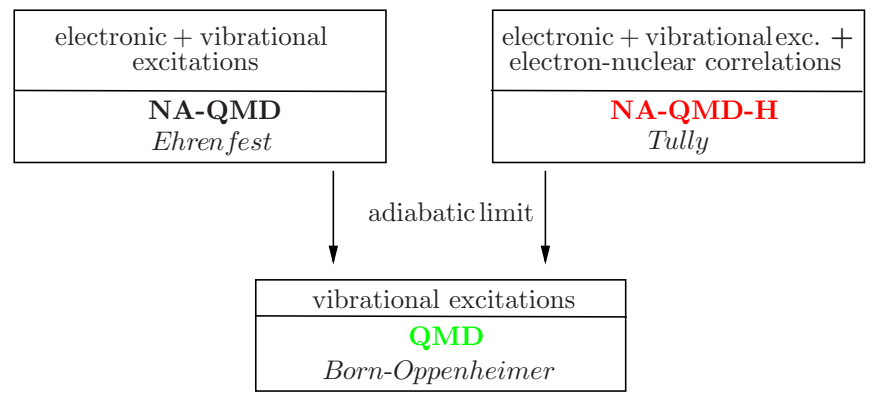

FIG. 1. (Color online) Overview of the $a b$ initio moleculardynamics methods used in this work: QMD, NA-QMD, and NAQMD-H. In the adiabatic limit (i.e., if electronic excitations do not occur in collisions) both nonadiabatic approaches (NA-QMD and NA-QMD-H) should merge into the QMD method (see the text and Paper I).

and the total fragmentation probability of the molecular target.

In Sec. III the theory will be confronted with an experiment of $\mathrm{He}+\mathrm{He}$ and $\mathrm{He}+\mathrm{H}_{2}$ collisions [41]. We will shed light on old, but still unexplained, experimental data of energy-loss spectroscopy, where highly differential observables were measured (e.g., the angular distribution of the projectile as a function of the energy loss). It will be shown that in this case ENCs are crucial in order to understand the measured spectra and that the present NA-QMD-H approach can explain them in detail.

In Sec. IV a brief summary and outlook are given.

Throughout this work the investigation will be performed by applying and comparing the results of three different $a b$ initio MD methods, which treat the collision dynamics of increasing generality (see Fig. 1 and Paper I for details):

(i) QMD, which considers a priori only vibrational excitations and thus describes the nuclear motion on the BornOppenheimer ground-state surface;

(ii) NA-QMD, which includes both vibrational and electronic excitations but treats the nuclear dynamics in the Ehrenfest limit; and

(iii) NA-QMD-H, which additionally takes into account ENC effects.

Comparing the results of the three approaches allows us to distinguish between initial electronic and vibrational excitations (QMD vs NA-QMD) and to reveal the relevance of ENC effects in excitation and relaxation (NA-QMD vs. NA-QMD-H). Evidentially, all three approaches should deliver identical results in the adiabatic limit, i.e., if electronic excitations do not occur.

\section{EXCITATION AND RELAXATION IN ATOM-MOLECULE AND ATOM-CLUSTER COLLISIONS}

In this section we investigate systematically atom-molecule and atom-cluster collisions with increasing complexity ranging from the diatomic one-electron case to the polyatomic many-electron case, namely, $\mathrm{He}+\mathrm{Na}_{2}{ }^{+}, \mathrm{He}+\mathrm{Na}_{2}, \mathrm{He}+\mathrm{N}_{2}$, and $\mathrm{He}+\mathrm{Na}_{9}{ }^{+}$collisions. It is the main aim to gain insight into the general relevance of ENCs on both the initial excitation process and the subsequent postcollisional relaxation mechanism under well-defined scattering conditions. Thus, for each system a fixed collisional geometry (with impact parameter zero) is considered whereas the impact energy is varied in a wide range. Details of all calculations, presented in this section, are summarized in Appendix 1.

First, the total kinetic energy loss $\Delta E$ (i.e., the total amount of electronic and vibrational excitation energy transferred to the molecular target) is investigated as a function of the impact velocity in all three MD variants. It is defined as

$$
\Delta E=E_{\text {c.m. }}-E_{\text {c.m. }}(t \rightarrow \infty),
$$

with $E_{\text {c.m. }}(t)=\frac{\mu}{2} v_{\text {rel }}(t)^{2}$, where $v_{\text {rel }}(t)$ is the relative velocity of the projectile and target and $\mu$ is their reduced mass. The impact energy is $E_{\text {c.m. }}=E_{\text {c.m. }}(t=0)$. Comparison of the results of the QMD calculations with those of the nonadiabatic methods (NA-QMD and NA-QMD-H) then clearly allows us to discriminate between the impulsive regime (where only rovibrational excitations occur), the electronic regime (where the excitation process is dominated by electronic transitions), and the intermediate transition region (where electronic and rovibrational excitations coexist). Evidently, the influence of ENCs on the initial excitation process can occur only in the electronic and transition regimes and will be quantified by comparing the $\Delta E$ values obtained from the NA-QMD and NA-QMD-H methods.

Second, the postcollisional relaxation process is studied by following the molecular dynamics up to time scales much longer than the collision time. As a quantitative measure of ENC effects, we consider in particular the fragmentation probability $P_{\mathrm{F}}$ in the whole range of impact velocities. Note that in the present academic QMD and NA-QMD studies (without orientation and impact parameter averaging), this quantity can only be exactly 1 or 0 (fragmentation or not) as the dynamics is described by a single deterministic trajectory for each impact velocity. This, however, must not be the case when ENCs are taken into account as the dynamics is described by an ensemble of stochastic trajectories at each impact velocity for one at the same initial condition.

For physical reasons, all three $a b$ initio MD formalisms must deliver identical results in the purely impulsive regime despite all very different equations of motion (see Paper I), which, by the way, may also serve as a sensitive test of the numerical implementations.

\section{A. $\mathrm{He}+\mathrm{Na}_{2}{ }^{+}$and $\mathrm{He}+\mathrm{Na}_{2}$ collisions}

The $\mathrm{He}+\mathrm{Na}_{2}{ }^{+}$system represents a one-electron system and has been investigated by us earlier $[15,42]$ under realistic scattering conditions (i.e., impact parameter and orientation averaged). Here it serves as reference system for the (as physically expected) similar $\mathrm{He}+\mathrm{Na}_{2}$ system, which represents an effective one-electron system (one active-electron pair).

The corresponding kinetic energy losses $\Delta E$ are shown in Figs. 2(a) and 2(b). Indeed, the $\mathrm{Na}_{2}{ }^{+}$and $\mathrm{Na}_{2}$ cases are qualitatively similar, apart from some quantitative differences. In both cases we are faced with two dominating regimes: For $E_{\text {c.m. }}<0.8 \mathrm{keV}$ the collision dynamics is governed by momentum transfer leading exclusively to rovibrational excitations (impulsive regime), whereas for impact energies $E_{\text {c.m. }}>2 \mathrm{keV}$ electronic excitations predominate (electronic regime). Appreciable simultaneous electronic and vibrational excitations occur in a rather small window of impact energies 

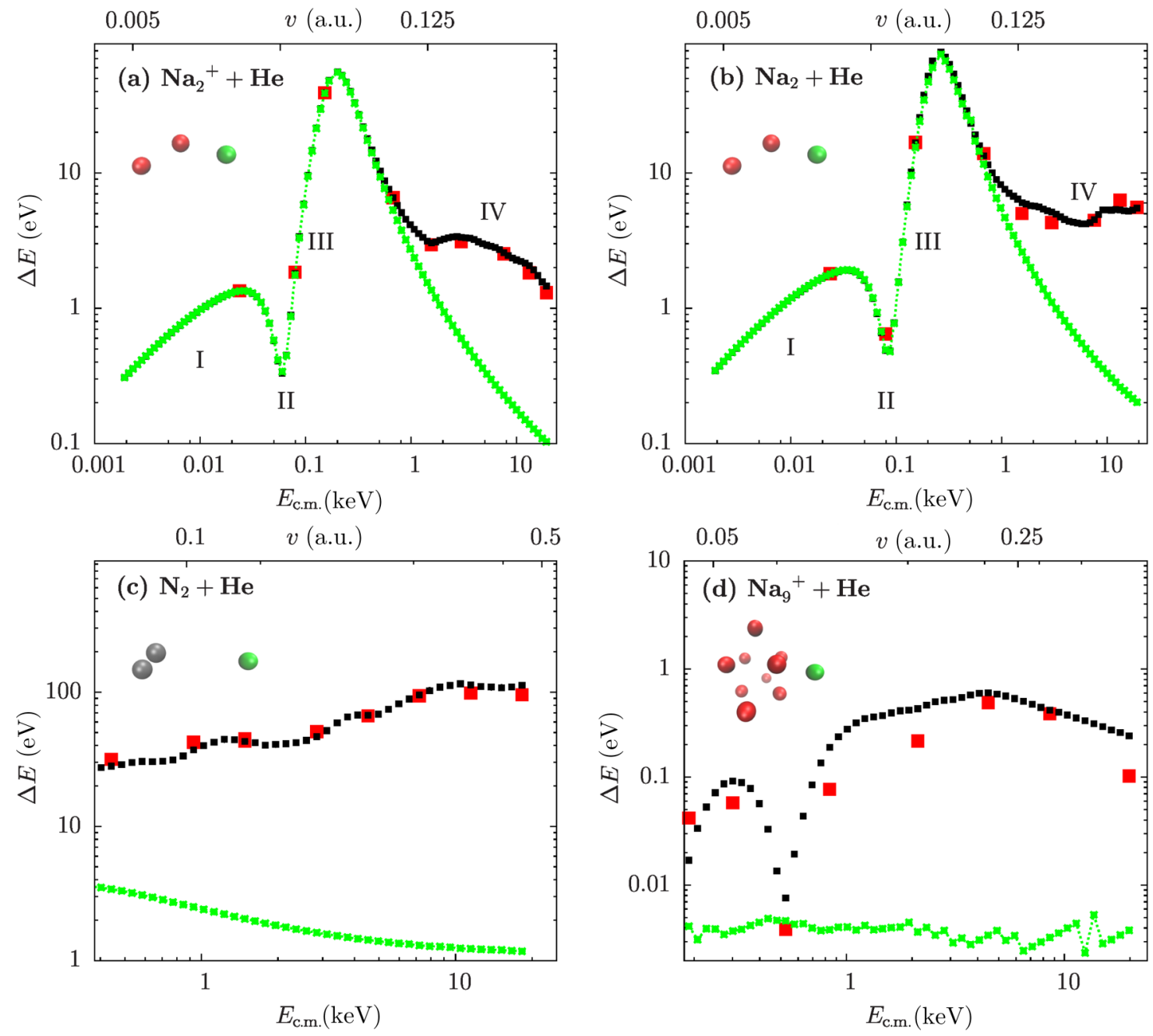

FIG. 2. (Color online) Kinetic energy loss $\Delta E$ as a function of the impact energy $E_{\text {c.m. }}$ for collisions of He with different targets calculated with QMD (green squares), NA-QMD (black squares), and NA-QMD-H (red squares): (a) $\mathrm{Na}_{2}{ }^{+}$, (b) $\mathrm{Na}_{2}$, (c) $\mathrm{N}_{2}$, and (d) $\mathrm{Na}_{9}{ }^{+}$. The top abscissae denote the corresponding impact velocities $v$. The collision geometries are sketched in the insets. The typical collision scenarios for the labeled impact energy regions I-IV in (a) and (b) are discussed in detail in the text.

located around $E_{\mathrm{c} . \mathrm{m} .}=1 \mathrm{keV}$ (transition regime). As expected, all three methods-QMD, NA-QMD and NA-QMD$\mathrm{H}$ - give exactly the same results in the impulsive regime, demonstrating nicely that the nonadiabatic MD approaches automatically merge into the adiabatic QMD limit (Fig. 1). More importantly, in the transition and electronic regimes, there is also no remarkable difference between the NAQMD and NA-QMD-H results, demonstrating that quantum effects in the nuclear dynamics do not affect the initial electronic excitation process. Maybe this is not too surprising as the wavelength of the projectile is orders of magnitude smaller than the scattering region (internuclear distance of the molecule) and thus a purely classical description of the scattering process is justified.

This, however, holds in the whole region of the considered impact energies in Fig. 2 and fairly well allows us to characterize the collision dynamics by single classical trajectories. By doing so, one can understand and interpret the general behavior of $\Delta E$ in Figs. 2(a) and 2(b) [increasing energy loss in region I, minimum in region II, maximum in region III, and plateau in region IV; see Figs. 2(a) and 2(b)] in full microscopic detail, discussed in the laboratory frame in the following.

(I) The $\mathrm{He}$ atom is scattered dominantly by the front $\mathrm{Na}$ atom with a scattering angle below $90^{\circ}$ (forward scattering with reflection).

(II) The $\mathrm{He}$ atom is scattered by both $\mathrm{Na}$ atoms with a similar effect with a scattering angle below $90^{\circ}$ (forward scattering with reflection), resulting in a translation of the target as a whole and minimum rovibrational excitation.

(III) The He atom is scattered by both $\mathrm{Na}$ atoms, but dominantly by the rear $\mathrm{Na}$ atom with a scattering angle above $90^{\circ}$ (backscattering). With increasing $E_{\text {c.m. }}$, in this range, a direct hit of both target atoms is possibly connected with a zigzag scattering through the target molecule, which leads to the maximum in the energy loss in Figs. 2(a) and 2(b) (passing through).

(IV) At high impact energies, the He atom passes through the target molecule nearly on a straight line without leaving significant vibrational excitations anymore.

These scenarios are observed in all three types of calculations. This fact, together with the same $\Delta E$ values in NA-QMD 

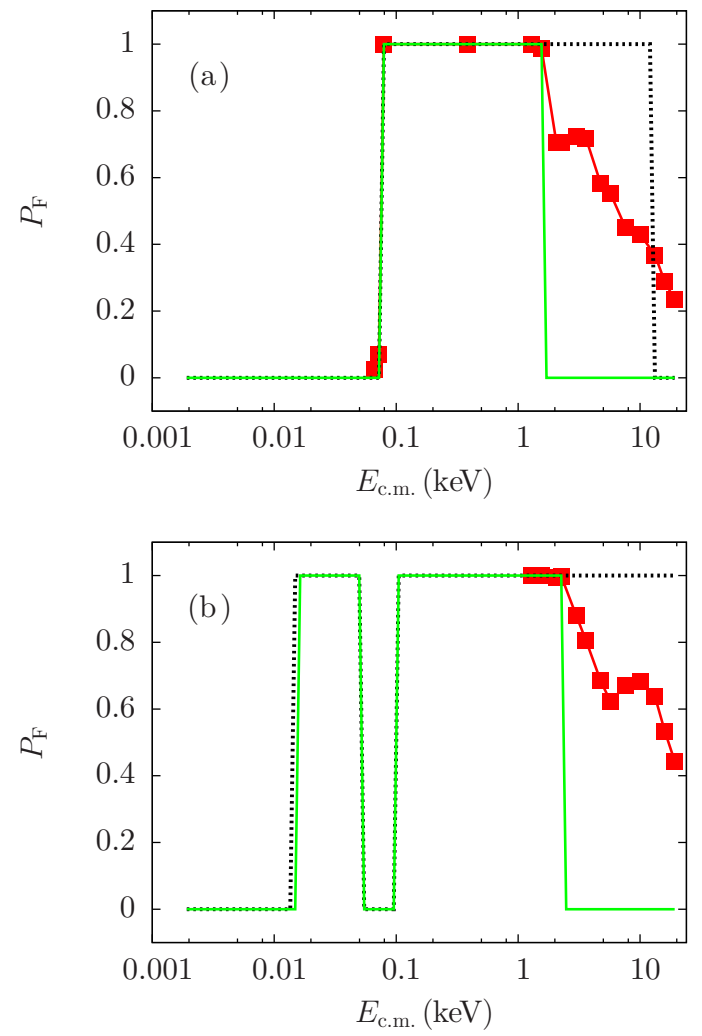

FIG. 3. (Color online) Fragmentation probability $P_{\mathrm{F}}$ as a function of the impact energy $E_{\text {c.m. }}$ for the same collisions as shown in Fig. 2: (a) He with $\mathrm{Na}_{2}{ }^{+}$and (b) $\mathrm{Na}_{2}$, from QMD (green squares), NA-QMD (black squares), and NA-QMD-H (red squares).

and NA-QMD-H calculations in Fig. 2, leads to the preliminary conclusion that ENCs do not affect the dynamics of the atomic projectile and/or the initial excitation process at least as long as integral quantities such as $\Delta E$ are considered.

Electron-nuclear correlation effects, however, arise distinctly in the postcollisional relaxation of the molecular target. In Fig. 3 the corresponding fragmentation probabilities are shown for both collisions. Again, all three methods predict nicely the same results in the pure impulsive regime, with $P_{\mathrm{F}}=1$ or 0 , as the (purely vibrational) excitation energy $\Delta E$ is larger or smaller than the fragmentation threshold of about $1 \mathrm{eV}$ (cf. ranges of $P_{\mathrm{F}}=1$ values in Fig. 3 and the $\Delta E$ values within the corresponding impact energy windows in Fig. 2).

In the transition and electronic regimes, the range of impact energies with $P_{\mathrm{F}}=1$ values is definitely larger in the NA-QMD calculations than for the QMD results. This is due to the fact that the total (vibrational and now also electronic) excitation energies exceed the fragmentation threshold and thus electron-vibration coupling leads to and allows for fragmentation, as it should. Nevertheless, the unphysical jumps of $P_{\mathrm{F}}$ from one to zero are inherent in the Ehrenfest dynamics of the nuclei as (at fixed initial conditions, i.e., without impact parameter and orientation averaging) the nuclear motion is described by one deterministic trajectory with only one fragmentation pathway.

This, however, is basically different if ENCs are taken into account and many fragmentation pathways (contained in the exact quantum-mechanical wave packet) are taken into account approximately. Clearly shown in Fig. 3, the fragmentation probabilities $P_{\mathrm{F}}$ (physically reasonable) smoothly decrease from 1 towards 0 above an $E_{\text {c.m. }}$. of about $2 \mathrm{keV}$, i.e., in the transition and purely electronic regimes. Thereby, they reflect all details of the structures in the $\Delta E$ values in Figs. 2(a) and 2(b), e.g., the plateau between 3 and $5 \mathrm{keV}$ in $\mathrm{He}+\mathrm{Na}_{2}{ }^{+}$ collisions [see Figs. 2(a) and 3(a)] or the maximum around $10 \mathrm{keV}$ in the $\mathrm{He}+\mathrm{Na}_{2}$ collisions [see Figs. 2(b) and 3(b)].

Concluding this part, ENCs are of vanishing importance in identifying the mean kinetic energy loss of the atomic projectile (excitation), but are relevant in describing realistically the subsequent relaxation of the molecular target (fragmentation), at least in simplest atom-molecule collisions, as investigated so far. This conclusion, however, remains unchanged if much more complex systems are considered, as will be shown in the following section.

\section{B. $\mathrm{He}+\mathrm{N}_{2}$ collision}

As our first "real" many-electron problem, collisions between a $\mathrm{He}$ atom (with frozen-core electrons) and a $\mathrm{N}_{2}$ target (with five active-electron pairs) will be considered. The total kinetic energy loss $\Delta E$ as a function of the impact energy $E_{\text {c.m. }}$ is shown in Fig. 2(c). The chosen interval of impact energies $(0.1-20 \mathrm{keV})$ is now restricted to the electronic regime [see vanishing $\Delta E$ values predicted by the QMD calculations in Fig. 2(c)], as in this region quantum effects in the nuclear dynamics can be inherently of importance (as long as they are taken into account approximately by the surface hopping methodology). Again, NA-QMD and NA-QMD-H results agree totally and thus ENCs are of unimportance for the global quantity total kinetic energy loss $\Delta E$ of the projectile.

To characterize the postcollisional dynamics, in Fig. 4 typical trajectories are shown as a function of time as obtained from the NA-QMD calculations. At the low impact energy $E_{\text {c.m. }}=1.5 \mathrm{keV}$ the excitation energy $\Delta E$ is still too small

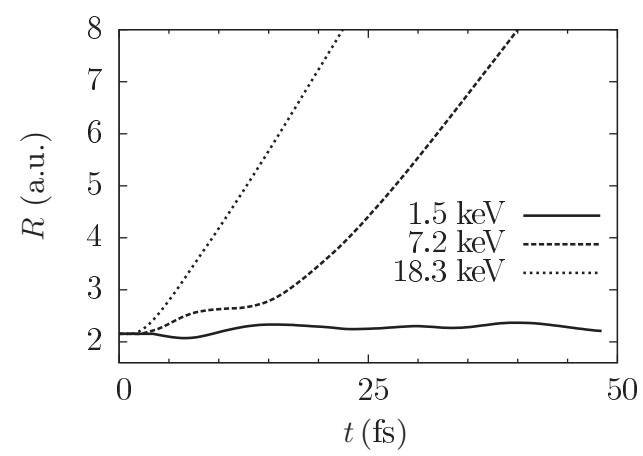

\begin{tabular}{|c|c|c|c|}
\hline \multirow{2}{*}{$E_{\text {c.m. }}(\mathrm{keV})$} & \multirow{2}{*}{$\begin{array}{c}\Delta E(\mathrm{eV}) \\
\text { NA-QMD-H }\end{array}$} & \multicolumn{2}{|c|}{$P_{\mathrm{F}}$} \\
\hline & & NA-QMD & NA-QMD-H \\
\hline 1.5 & 43 & 0 & 0.38 \\
\hline 7.2 & 94 & 1 & 0.50 \\
\hline 18.3 & 96 & 1 & 0.61 \\
\hline
\end{tabular}

FIG. 4. The top panel shows the internuclear distance $R$ of $\mathrm{N}_{2}$ calculated as a function of time $t$ in NA-QMD for three selected impact energies $E_{\text {c.m. }}$. The bottom panel shows the excitation energies $\Delta E$ [cf. Fig. 2(c)] and fragmentation probabilities $P_{\mathrm{F}}$ predicted by the NA-QMD and NA-QMD-H methods. 
to fragment the molecule and electron-vibration coupling induces only vibrations, whereas at larger impact energies $E_{\text {c.m. }}=7.2$ and $18.3 \mathrm{keV}$ fragmentation occurs. Interestingly, the fragmentation dynamics is different in both cases with delayed fragmentation for $E_{\text {c.m. }}=7.2 \mathrm{keV}$ and immediate decay for $E_{\text {c.m. }}=18.3 \mathrm{keV}$, although the $\Delta E$ values are nearly equal [cf. Fig. 2(c)]. From this different dynamics one may intuitively expect an influence on the fragmentation probabilities $P_{\mathrm{F}}$ as predicted by the NA-QMD-H calculations. This is indeed the case, as shown in the table of the lower part in Fig. 4, together with the $\Delta E$ values. While the increase of $P_{\mathrm{F}}$ between $E_{\text {c.m. }}=1.5$ and $7.2 \mathrm{keV}$ is not very surprising, the further distinct increase at $E_{\mathrm{c} . \mathrm{m} .}=18.3 \mathrm{keV}$ is nontrivial as the $\Delta E$ values practically agree at $E_{\text {c.m. }}=7.2$ and $18.3 \mathrm{keV}$. This clearly demonstrates that the total amount of electronic excitation energy is not enough to describe the fragmentation process, which is of basically nonstatistical nature.

\section{C. $\mathrm{He}+\mathrm{Na}_{9}{ }^{+}$collision}

The $\mathrm{He}+\mathrm{Na}_{9}{ }^{+}$collision has been studied in full detail using the NA-QMD approach [38]. Here it is of special interest to investigate to what extent ENC effects may possibly modify the general conclusions drawn in [38].

The corresponding kinetic energy losses $\Delta E$ are shown in Fig. 2(d) again in the electronic regime (see vanishing $\Delta E$ values in the QMD calculations in the figure). In addition, the chosen collisional geometry allows for absolute $\Delta E$ values that are distinctly smaller than in the previous cases and thus hopefully more sensitive against possible ENC effects. Indeed, there are now distinct quantitative differences between the $\Delta E$ values calculated in the NA-QMD and the NA-QMD-H methods. Qualitatively, however, the results nicely agree and the small quantitative differences will be of negligible relevance if orientation and impact parameter averaging is taken into account to predict the final experimental (integral) $\Delta E$ values. In particular, the distinct minimum at certain impact energy (around $E_{\text {c.m. }} \approx 0.5 \mathrm{keV}$ ) with practically vanishing energy losses is nicely reproduced in both calculations. It is just this peculiarity that leads to the so-called transparency effect, predicted for this collisional system [38].

\section{ENERGY-LOSS-ANGLE CORRELATIONS IN $\mathrm{He}+\mathrm{H}_{2}$ AND He + He COLLISIONS: COMPARISON WITH EXPERIMENT}

Following the previous integral studies of the gross features of ENC effects in atomic collisions, in this section we confront the theory with experiment, taking into account all impact parameters and many molecular orientations in $\mathrm{He}+\mathrm{H}_{2}$ and $\mathrm{He}+\mathrm{He}$ collisions at fixed laboratory impact energies of $E_{\text {lab }}=2 \mathrm{keV}$ (corresponding to a center-of-mass velocity of $v=0.14$ a.u.) and $E_{\text {lab }}=1 \mathrm{keV}$ (corresponding to $v=$ 0.1 a.u.), respectively. For these systems, highly differential quantities have been measured in experiments using energyloss spectroscopy [41]. Energy-loss spectroscopy represents an advanced experimental collision technique, which allows one to obtain detailed insight into the reaction mechanism, e.g., to distinguish between vibrational and electronic excitations, or to investigate differential charge transfer and electron-capture cross sections $[41,43]$.
In particular, the total kinetic energy loss of the He projectile as a function of the scattering angle has been measured [41]. Although quantitatively different, the results are qualitatively very similar in both systems, exhibiting three characteristic peaks in the laboratory energy-loss spectra [41]. Thereby, the first peak, located at smallest energy losses, has been interpreted as originating from electronically elastic collisions. The second and third peaks, located at distinctly higher energy losses, were attributed to one- and two-electron transitions, respectively. Thereby, in the $\mathrm{He}+\mathrm{He}$ collisions, the transitions are $a$ priori induced in both colliding $\mathrm{He}$ atoms and the $\mathrm{He}+\mathrm{H}_{2}$ collisions, expected to occur preferably [41] within the molecular target $\mathrm{H}_{2}$ only. A quantitative and dynamical analysis of the experimental findings and their interpretation will be presented below.

In the next section the theoretical foundations and approximations (necessary to treat realistically these complex collision systems) are summarized and the electronic excitation energies of $\mathrm{He}$ and $\mathrm{H}_{2}$ are discussed (essential to interpret the results). All technical and numerical details of the calculations are summarized in Appendix 2.

\section{A. Restricted Hartree-Fock theory with the half-electron approximation}

In order to treat explicitly one-electron excitations as well as to discriminate between one- and two-electron excitations requires, in principle, a spin-unrestricted many-body approach, e.g., local spin-density approximation or spin-unrestricted Hartree-Fock (UHF) theory. In both methods, however, one is faced with the spin-contamination problem [44], which may cause severe problems in the adiabatic state tracking procedure [45] that is indispensable, however, in the NA-QMD-H calculations. To avoid this serious problem and in order to describe realistically electronic excitations in the $\mathrm{He}$ atom, we propose and apply here a restricted Hartree-Fock method within the so-called half-electron (RHF-HE) approximation in the spirit of [46].

Originally, the half-electron idea was used to allow for the description of open-shell systems, such as hydrocarbon radicals and radical ions, using a modified closed-shell, i.e., spin-restricted, method. In its heart, the unpaired electron of the radical is replaced by two half electrons of opposite spin [46]. Here we extend this approach by replacing all electron pairs by two half-electron pairs, which are treated as if they were electrons of opposite spin. With this mathematical trick we are able to account, approximately, for single-electron transitions and avoid any spin-contamination problem.

The energy-level diagrams corresponding to both RHF-HE and UHF calculations are shown and compared in Fig. 5. In both cases, the energy levels separate into two distinct regions: one-electron (I) and two-electron excitations (II). As can be seen in Fig. 5, the RHF-HE approximation merely leads to a small shift of the excitation energies as compared to the more accurate UHF energies and thus provides a reasonable description of the excitation spectrum. Note in advance that the doubly excited states in $\mathrm{H}_{2}$ are located at distinctly lower excitation energies as compared to that within the He atom and, in addition, are clearly more dense. 

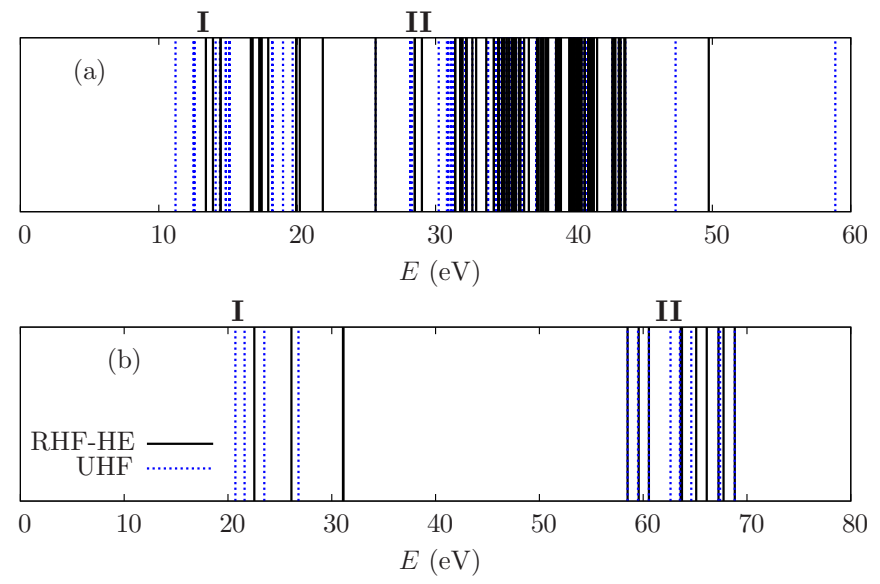

FIG. 5. (Color online) Excitation energies, constructed from bare HF one-electron excitations (I) and double-electron (II) excitations calculated using the RHF-HE and UHF methods for (a) the $\mathrm{H}_{2}$ molecule at its equilibrium internuclear distance and (b) the $\mathrm{He}$ atom.

\section{B. Results}

As the central quantity, we calculate the kinetic energy loss $Q$ of the He projectile in dependence on the scattering angle $\theta$ in the laboratory frame defined as

$$
Q(\theta)=\frac{M_{\mathrm{He}}}{2}\left[v_{\mathrm{He}}^{2}(t=0)-v_{\mathrm{He}}^{2}(t \rightarrow \infty, \theta)\right] .
$$

Physically, it is the same quantity as defined in (1), but angularly resolved and now considered in the laboratory frame, according to the experimental data [41].

The calculated spectra for the $\mathrm{He}+\mathrm{He}$ collisions are shown in Figs. 6(a) and 6(c). The QMD and the NA-QMD results [Fig. 6(a)] exhibit a one-to-one correspondence of energy loss $Q$ and scattering angle $\theta$ as one could expect from the unambiguity between scattering angle $\theta$ and impact parameter $b$ enabling also a very transparent interpretation: The largest impact parameters result in completely elastic collisions and thus vanishing $\theta$. With decreasing $b$ values the resting target atom is more frequently encountered, leading to increasing deflection connected with increasing energy loss in both cases. Thereby, the purely kinematic energy loss in the laboratory system is reflected by the QMD data, whereas additional electronic excitations lead to pronounced larger- $Q$ values in the NA-QMD calculations up to maximum measured values of $Q \approx 60 \mathrm{eV}$. A widespread angular distribution (as measured for a fixed energy loss $Q$ ), however, cannot be basically obtained in atom-atom collisions, in either (deterministic Born-Oppenheimer and Ehrenfest) methods.

The situation is fundamentally changed if ENCs are taken into account. In Fig. 6(c) the spectrum is shown as resulting from the NA-QMD-H theory and compared with experiment. As can be seen clearly, there are three bunches in the theoretical distribution (red squares), located between $Q$ values of 0 and about $10 \mathrm{eV}, 25$ and $35 \mathrm{eV}$, and 50 and $60 \mathrm{eV}$,
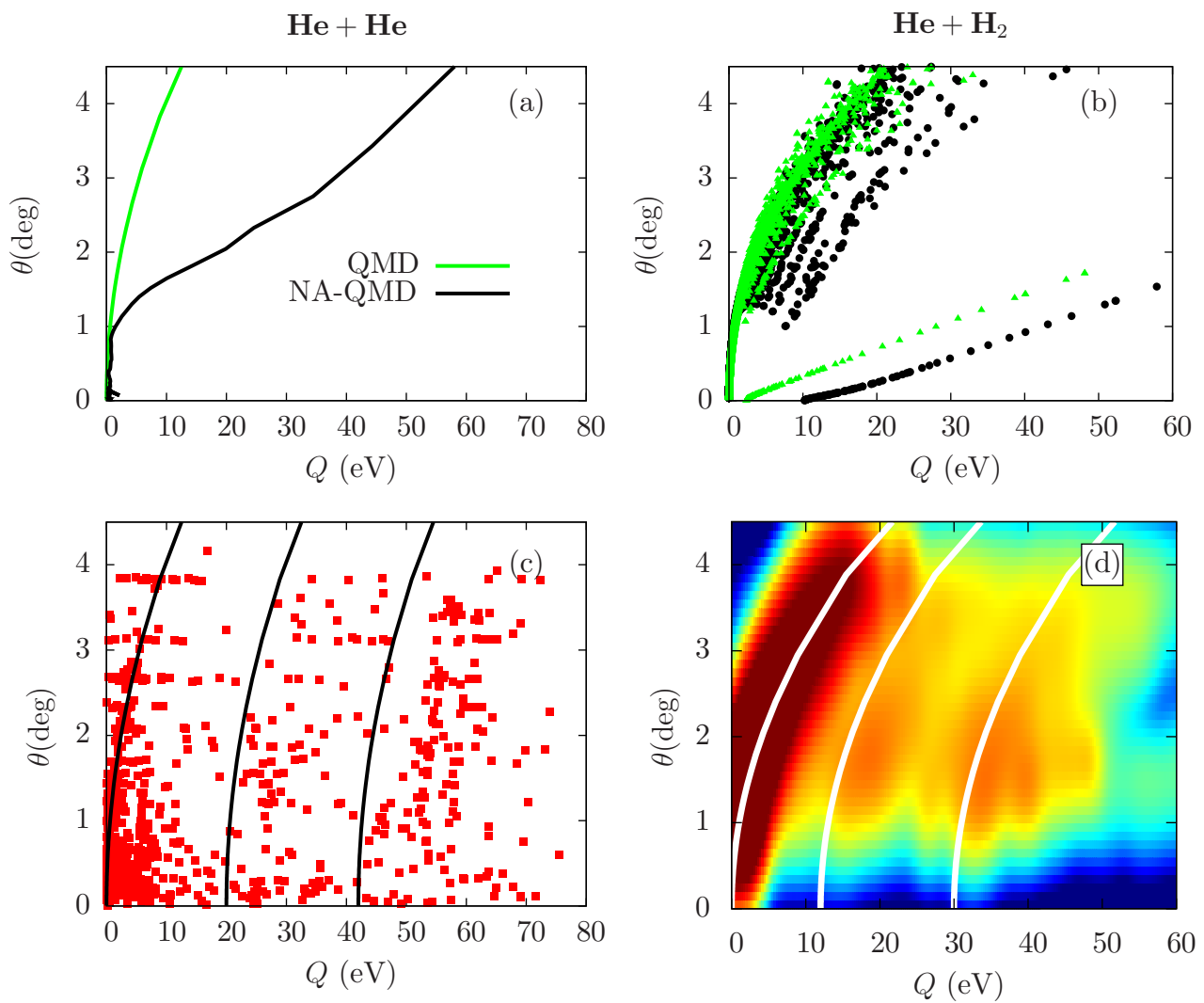

FIG. 6. (Color online) Angle-resolved energy-loss spectra for the (a) and (c) $\mathrm{He}+\mathrm{He}$ and (b) and (d) $\mathrm{He}+\mathrm{H}_{2}$ collisions: (a) and (b) results calculated with QMD (green) and NA-QMD (black) and (c) and (d) NA-QMD-H spectra represented (c) by red squares and (d) as a density plot by smoothing the results with localized Gaussian functions. The colors of the density plot range from blue (zero probability) to red (maximum probability). In addition, the black and white lines in (c) and (d), respectively, show experimental peak positions extracted from [41]. 
respectively. The first bunch agrees perfectly with experiment and is kinematically dominated [cf. the green QMD curve in Fig. 6(a) with the experimental points in Fig. 6(b)]. The fluctuations in the calculations show, however, that also in this region electronic transitions together with ENCs influence the spectra. The other two bunches, dominated and generated mainly by one- and two-electron excitations, respectively, are shifted by about $10 \mathrm{eV}$ to higher- $Q$ values as compared to experiment. This originates from the HF approximation, which does not account for the correlations needed, however, to describe the excitation energies in He with high precision. Nevertheless, the NA-QMD-H calculations reflect nicely the experimental three-peak structure and, together with the QMD and NA-QMD simulations, explain it in detail.

Finally, we consider the molecular collision system $\mathrm{He}+$ $\mathrm{H}_{2}$ for which the results are presented in Figs. 6(b) and 6(d). In this case, the QMD and NA-QMD calculations exhibit inherently also fluctuations in the $Q(\theta)$ distribution resulting naturally from different initial orientations of the molecular target [Fig. 6(a)]. There are two noticeable well separated regions of events, the first one at relatively-low- $Q$ values and large angles (with statistically distributed points) and the second one at large- $Q$ values and small angles (with regularly arranged points), in both calculations. Evidently, the second branch results from very small impact parameters and almost one specific orientation resulting in a maximum impulsive energy transfer (cf. also the discussion of trajectories in Sec. II A). These exotic events, however, can hardly be identified experimentally as they are connected with the smallest impact parameters $b \approx 0$ and thus vanishing weight in the experimental spectra. The interpretation of the first region is basically the same as in the former $\mathrm{He}+\mathrm{He}$ case. Notably, however, the absolute values of the electronic contribution to the total kinetic energy losses $Q$ are distinctly smaller as compared to $\mathrm{He}+\mathrm{He}$ collisions, which, on the other side, could also be intuitively expected from the differences in the electronic excitation spectra of $\mathrm{He}$ and $\mathrm{H}_{2}$ in Fig. 5 (note again here that we take into account electronic excitations only in the molecular $\mathrm{H}_{2}$ target). Although there exist indeed some regular structures in the NA-QMD distribution, resulting apparently from specific orientations, they have nothing to do with the three-peak structure of the experimental results, as shown in Fig. 6(d), where also the NA-QMD-H predictions are shown. Obviously, the NA-QMD-H theory reproduces quantitatively the experimental findings and confirms the given qualitative interpretation of the spectra in terms of impulsive transfer as well as one and two electronic transition mechanisms, as expected [41]. More generally and importantly, the present study demonstrates that ENC effects (i.e., quantum effects in the relative motion in high energetic atomic collisions with de Broglie wavelengths much smaller than the interaction region) become noticeable even in the initial electronic excitation process.

\section{SUMMARY AND OUTLOOK}

We presented a systematic application of the recently developed NA-QMD-H method (paper I) to atomic collisions with complex targets. In particular, the role of ENC effects in the excitation and relaxation processes has been examined by means of a comparative study utilizing three different $a b$ initio MD methods of increasing generality, i.e., QMD, NA-QMD, and NA-QMD-H. We found the following.

(i) As long as integral quantities are considered (mean total kinetic energy loss $\Delta E$ ), ENC effects are unimportant for a realistic description of the excitation process.

(ii) The relaxation mechanism in molecular targets, however, can be reasonably described physically and understood only if ENCs are taken into account (total fragmentation probability $P_{\mathrm{F}}$ for a given impact parameter and a selected orientation). To what extent this conclusion will be modified if impact parameter and orientation averaged collisions (as usually realized in experiments) are considered remains an interesting question for future studies.

(iii) If highly differential quantities are considered (total kinetic energy loss as a function of the scattering angle) quantum effects in the relative motion of the nuclei (approximated by surface hopping) appear massive and must be taken into account to understand and interpret the experimental findings, particularly in collisions where the de Broglie wavelength is much smaller than the interaction region (and thus a purely classical description of the relative motion seems to be appropriate).

(iv) We have developed a many-body technique, the RHF-HE approximation, which helps to avoid the spincontamination problem.

Finally, the very good agreement with experimental energyloss spectra demonstrates the predictive power of the present NA-QMD-H theory. In [47] we investigate excitation and relaxation (isomerization) mechanisms of photoexcited organic molecules.

\section{ACKNOWLEDGMENTS}

We gratefully acknowledge the allocation of computer resources from the ZIH of the Technische Universität Dresden and the financial support from the Deutsche Forschungsgemeinschaft through the Normalverfahren.

\section{APPENDIX: DETAILS OF THE CALCULATIONS}

\section{Atom-molecule and atom-cluster collisions}

In Sec. II we presented a systematic study of atom-molecule and atom-cluster collisions, focusing on excitations in the target molecule $\left(\mathrm{Na}_{2}{ }^{+}, \mathrm{Na}_{2}\right.$, and $\left.\mathrm{N}_{2}\right)$ or cluster $\left(\mathrm{Na}_{9}{ }^{+}\right)$. Therefore, we only take into account the valence electrons of $\mathrm{Na}$ and $\mathrm{N}$ explicitly in the calculations, while the remaining electrons, in particular those of the He projectile, are treated using the frozen-core approximation.

For all collisions, the optimized atomic basis sets are constructed from Gaussian basis sets [48-50] using an additional attractive potential $V(r)=\left(\frac{r}{r_{0}}\right)^{2}$ [51,52], which describes a pseudoatom and gives a better description of the molecular properties [53]. The details of the basis sets are summarized in Table I. The electronic density is described in all cases using the deMon Coulomb fitting basis set ( $s, p$-type Gaussian functions only).

The calculations in Sec. II (QMD, NA-QMD, and NA-QMD-H) are based on spin-restricted (time-dependent) 
TABLE I. Atomic basis set details: explicitly considered atomic orbitals (i.e., not treated using the frozen-core approximation), fundamental Gaussian basis sets, and pseudoatom parameters $r_{0}$.

\begin{tabular}{lccc}
\hline \hline Atom & Atomic orbitals & Gaussian basis set & $r_{0}$ (a.u.) \\
\hline $\mathrm{He}$ & $2 s$ & $6-31 \mathrm{G}$ & 3.5 \\
$\mathrm{Na}$ & $3 s, 3 p$ & $6-31 \mathrm{G}$ & 5.8 \\
$\mathrm{~N}$ & $2 s, 2 p, 3 s, 3 p$ & d-aug-cc-pV6Z $(s, p$ only $)$ & 1.9 \\
\hline \hline
\end{tabular}

density-functional theory using the adiabatic local-density approximation [54,55] for the exchange-correlation potential in the case of the many-electron targets $\left(\mathrm{Na}_{2}, \mathrm{~N}_{2}\right.$, and $\left.\mathrm{Na}_{9}{ }^{+}\right)$.

The collision scenarios are set as follows. Initially, the system is in the electronic ground state and the target molecules are in their ground-state equilibrium geometry with a fixed initial orientation with respect to the collision axis. We mention that the respective equilibrium internuclear distances of the target molecules in our calculations are 6.9 a.u. for $\mathrm{Na}_{2}{ }^{+}$, 6.4 a.u. for $\mathrm{Na}_{2}$, and 2.2 a.u. for $\mathrm{N}_{2}$, whereas the cluster radius for $\mathrm{Na}_{9}{ }^{+}$is 6.6 a.u. The He atom starts 20 a.u. away from the molecular target with impact parameter $b=0$ a.u., i.e., we consider only central collisions.

While single deterministic trajectories for each impact velocity are obtained in the QMD and NA-QMD calculations, the NA-QMD-H results for the kinetic energy loss $\Delta E$ or the fragmentation probability $P_{\mathrm{F}}$ are averaged over 250 trajectories for molecular targets $\left(\mathrm{Na}_{2}{ }^{+}, \mathrm{Na}_{2}\right.$, and $\left.\mathrm{N}_{2}\right)$ and 100 trajectories for $\mathrm{Na}_{9}{ }^{+}$targets for each impact energy. We note that a trajectory is considered to be fragmented if the internuclear distance $R$ exceeds $R_{\mathrm{F}}=12.8$ a.u. for $\mathrm{Na}_{2}{ }^{+}$and $R_{\mathrm{F}}=12.4$ a.u. for $\mathrm{Na}_{2}$ within a propagation time of $500 \mathrm{fs}$ and $R_{\mathrm{F}}=8$ a.u. for $\mathrm{N}_{2}$ within a propagation time of $50 \mathrm{fs}$.

\section{2. $\mathrm{He}+\mathrm{He}$ and $\mathrm{He}+\mathrm{H}_{2}$ collisions}

In Sec. III we confronted theoretical with experimental energy-loss spectra for the $\mathrm{He}+\mathrm{He}$ and $\mathrm{He}+\mathrm{H}_{2}$ collisions. In the latter case, excitations in the $\mathrm{H}_{2}$ target are expected to
TABLE II. Atomic basis set details for the $\mathrm{He}+\mathrm{H}_{2}$ and $\mathrm{He}+\mathrm{He}$ collisions: explicitly considered atomic orbitals (i.e., not treated using the frozen-core approximation), fundamental Gaussian basis sets, and pseudoatom parameters $r_{0}$.

\begin{tabular}{lcccc}
\hline \hline Collision & Atom & Atomic orbitals & Gaussian basis set & $\begin{array}{c}r_{0} \\
(\text { a.u. })\end{array}$ \\
\hline $\mathrm{He}+\mathrm{H}_{2}$ & $\mathrm{H}$ & $1 s, 2 s, 2 p, 3 s, 3 p$ & d-aug-cc-pV6Z & \\
& & & $(s, p$ only $)$ & \\
& $\mathrm{He}$ & $2 s$ & $6-31 \mathrm{G}$ & 3.5 \\
$\mathrm{He}+\mathrm{He}$ & $\mathrm{He}$ & $1 s, 2 s, 2 p, 3 s, 3 p$ & d-aug-cc-pV5Z & 3.5 \\
& & $1 s, 2 s, 2 p, 3 s, 3 p$ & $(s, p$ only $)$ & 3.5 \\
\hline \hline
\end{tabular}

dominate the spectra [41] and thus we treat the electrons of the He projectile within the frozen-core approximation for the $\mathrm{He}+\mathrm{H}_{2}$ collision.

The atomic basis sets for the respective atoms are summarized in Table II. As in Appendix 1, these optimized atomic basis sets are constructed from Gaussian basis sets [48-50], where we use an additional attractive (pseudoatom) potential $V(r)=\left(\frac{r}{r_{0}}\right)^{2}$ for the He atom for both collisions. The electronic density is described in all cases using the deMon Coulomb fitting basis set ( $s, p$-type Gaussian functions only).

All calculations in Sec. III (QMD, NA-QMD, and NA-QMD-H) are based on spin-restricted (time-dependent) Hartree-Fock theory using the half-electron approximation in the NA-QMD-H calculations (see Sec. III A).

The collision scenarios are set as follows. Initially, the system is in the electronic ground state. For both collisions the impact parameters $b$ are chosen from $b=0$ to 4 a.u. For the molecular target initial orientations were chosen randomly, thereby keeping the initial internuclear distance fixed at its ground-state equilibrium value. While single, deterministic trajectories for each impact parameter and orientation results from the QMD and NA-QMD calculations, trajectories are sampled stochastically for the NA-QMD-H calculations. The NA-QMD-H results for the angle-resolved energy-loss spectra are obtained from $\sim 10^{4}$ trajectories for the $\mathrm{He}+\mathrm{He}$ collision and from $\sim 10^{5}$ trajectories for the $\mathrm{He}+\mathrm{H}_{2}$ collision.
[1] A. Kis, G. Csanyi, J.-P. Salvetat, T.-N. Lee, E. Couteau, A. J. Kulik, W. Benoit, J. Brugger, and L. Forro, Nat. Mater. 3, 153 (2004).

[2] C. Gömez-Navarro, P. J. De Pablo, J. Gómez-Herrero, B. Biel, F. J. Garcia-Vidal, A. Rubio, and F. Flores, Nat. Mater. 4, 534 (2005).

[3] H. Stahl, J. Appenzeller, R. Martel, P. Avouris, and B. Lengeler, Phys. Rev. Lett. 85, 5186 (2000).

[4] P. Esquinazi, D. Spemann, R. Höhne, A. Setzer, K.-H. Han, and T. Butz, Phys. Rev. Lett. 91, 227201 (2003).

[5] S. Talapatra, P. G. Ganesan, T. Kim, R. Vajtai, M. Huang, M. Shima, G. Ramanath, D. Srivastava, S. C. Deevi, and P. M. Ajayan, Phys. Rev. Lett. 95, 097201 (2005).

[6] S. Zamith, P. Labastie, and J.-M. L'Hermite, J. Chem. Phys. 136, 214301 (2012).
[7] T. Schlathölter, O. Hadjar, R. Hoekstra, and R. Morgenstern, Phys. Rev. Lett. 82, 73 (1999).

[8] M. Ehrich, U. Werner, H. O. Lutz, T. Kaneyasu, K. Ishii, K. Okuno, and U. Saalmann, Phys. Rev. A 65, 030702 (2002).

[9] C. Bréchignac, P. Cahuzac, B. Concina, J. Leygnier, L. F. Ruiz, B. Zarour, P. A. Hervieux, J. Hanssen, M. F. Politis, and F. Martín, Phys. Rev. Lett. 89, 183402 (2002).

[10] X. Urbain, N. de Ruette, V. M. Andrianarijaona, M. F. Martin, L. F. Menchero, L. F. Errea, L. Méndez, I. Rabadán, and B. Pons, Phys. Rev. Lett. 111, 203201 (2013).

[11] S. Trippel, M. Stei, J. A. Cox, and R. Wester, Phys. Rev. Lett. 110, 163201 (2013).

[12] Z. Roller-Lutz, Y. Wang, H. O. Lutz, U. Saalmann, and R. Schmidt, Phys. Rev. A 59, R2555 (1999).

[13] F. Rohmund, E. E. B. Campbell, O. Knospe, G. Seifert, and R. Schmidt, Phys. Rev. Lett. 76, 3289 (1996). 
[14] H. Zettergren, P. Rousseau, Y. Wang, F. Seitz, T. Chen, M. Gatchell, J. D. Alexander, M. H. Stockett, J. Rangama, J. Y. Chesnel et al., Phys. Rev. Lett. 110, 185501 (2013).

[15] J. A. Fayeton, M. Barat, J. C. Brenot, H. Dunet, Y. J. Picard, U. Saalmann, and R. Schmidt, Phys. Rev. A 57, 1058 (1998).

[16] S. Martin, L. Chen, R. Brédy, G. Montagne, C. Ortega, T. Schlathölter, G. Reitsma, and J. Bernard, Phys. Rev. A 85, 052715 (2012).

[17] A. Lawicki, A. I. S. Holm, P. Rousseau, M. Capron, R. Maisonny, S. Maclot, F. Seitz, H. A. B. Johansson, S. Rosén, H. T. Schmidt et al., Phys. Rev. A 83, 022704 (2011).

[18] E. E. B. Campbell and F. Rohmund, Rep. Prog. Phys. 63, 1061 (2000).

[19] J. Opitz, H. Lebius, S. Tomita, B. A. Huber, P. Moretto Capelle, D. Bordenave Montesquieu, A. Bordenave Montesquieu, A. Reinköster, U. Werner, H. O. Lutz et al., Phys. Rev. A 62, 022705 (2000).

[20] Y. Nakai, T. Majima, T. Mizuno, H. Tsuchida, and A. Itoh, Phys. Rev. A 83, 053201 (2011).

[21] F. Calvayrac, P.-G. Reinhard, E. Suraud, and C. Ullrich, Phys. Rep. 337, 493 (2000).

[22] A. V. Krasheninnikov, Y. Miyamoto, and D. Tománek, Phys. Rev. Lett. 99, 016104 (2007).

[23] P. Lopez-Tarifa, M.-A. Herve du Penhoat, R. Vuilleumier, M.-P. Gaigeot, I. Tavernelli, A. Le Padellec, J.-P. Champeaux, M. Alcami, P. Moretto-Capelle, F. Martín et al., Phys. Rev. Lett. 107, 023202 (2011).

[24] A. Castro, M. Isla, J. I. Martinez, and J. A. Alonso, Chem. Phys. 399, 130 (2012).

[25] C.-Z. Gao, J. Wang, and F.-S. Zhang, Chem. Phys. 410, 9 (2013).

[26] U. Saalmann and R. Schmidt, Z. Phys. D 38, 153 (1996).

[27] T. Kunert and R. Schmidt, Eur. Phys. J. D 25, 15 (2003).

[28] M. Uhlmann, T. Kunert, and R. Schmidt, J. Phys. B 39, 2989 (2006).

[29] M. Uhlmann, T. Kunert, and R. Schmidt, Phys. Rev. E 72, 036704 (2005).

[30] J. C. Tully, J. Chem. Phys. 137, 22A301 (2012).

[31] D. Amit, Field Theory, the Renormalization Group, and Critical Phenomena (McGraw-Hill, New York, 1978).

[32] M. F. Politis, P. A. Hervieux, J. Hanssen, M. E. Madjet, and F. Martín, Phys. Rev. A 58, 367 (1998).
[33] P. A. Hervieux, B. Zarour, J. Hanssen, M. F. Politis, and F. Martín, J. Phys. B 34, 3331 (2001).

[34] Z.-P. Wang, P. M. Dinh, P. G. Reinhard, E. Suraud, and F. S. Zhang, Int. J. Quantum Chem. 111, 480 (2011).

[35] D. Babikov, E. Gislason, M. Sizun, F. Aguillon, and V. Sidis, J. Chem. Phys. 112, 9417 (2000).

[36] P. J. Kuntz, Atom-Molecule Collision Theory (Plenum, New York, 1979), p. 79.

[37] M. Fischer, J. Handt, and R. Schmidt, Paper I of this series, Phys. Rev. A 90, 012525 (2014).

[38] U. Saalmann and R. Schmidt, Phys. Rev. Lett. 80, 3213 (1998).

[39] T. Kunert and R. Schmidt, Phys. Rev. Lett. 86, 5258 (2001).

[40] T. Laarmann, I. Shchatsinin, A. Stalmashonak, M. Boyle, N. Zhavoronkov, J. Handt, R. Schmidt, C. P. Schulz, and I. V. Hertel, Phys. Rev. Lett. 98, 058302 (2007).

[41] D. Dowek, D. Dhuicq, V. Sidis, and M. Barat, Phys. Rev. A 26, 746 (1982).

[42] F. Grossmann, U. Saalmann, and R. Schmidt, Ann. Phys. (Leipzig) 9, 785 (2000).

[43] E. J. Quintana and E. Pollack, Phys. Rev. A 57, 1448 (1998).

[44] A. Szabó and N. Ostlund, Modern Quantum Chemistry: Introduction to Advanced Electronic Structure Theory (Dover, New York, 1989).

[45] L. F. Errea, L. Fernandez, A. Macias, L. Mendez, I. Rabadan, and A. Riera, J. Chem. Phys. 121, 1663 (2004).

[46] M. J. S. Dewar, J. A. Hashmall, and C. G. Venier, J. Am. Chem. Soc. 90, 1953 (1968).

[47] M. Fischer, J. Handt, and R. Schmidt, Paper III of this series, Phys. Rev. A 90, 012527 (2014).

[48] Extensible computational chemistry environment basis set database, http://bse.pnl.gov/bse/portal.

[49] D. Feller, J. Comput. Chem. 17, 1571 (1996).

[50] K. L. Schuchardt, B. T. Didier, T. Elsethagen, L. Sun, V. Gurumoorthi, J. Chase, J. Li, and T. L. Windus, J. Chem. Inf. Model. 47, 1045 (2007).

[51] H. Eschrig and I. Bergert, Phys. Status Solidi B 90, 621 (1978).

[52] H. Eschrig, Optimized LCAO Method and the Electronic Structure of Extended Systems (Springer, Berlin, 1989).

[53] T. Kunert, Ph.D. thesis, TU Dresden, 2003.

[54] A. Zangwill and P. Soven, Phys. Rev. Lett. 45, 204 (1980).

[55] A. Zangwill and P. Soven, Phys. Rev. B 24, 4121 (1981). 